\title{
A Novel VPON Scheme Syncretizing Multiple Subsystems for Metro-Access Optical Network
}

\author{
Weidong Xia, Chaoqin Gan, Weilun Xie, Shucong Ma, Wei Li \\ Key Laboratory of Specialty Fiber Optics and Optical Access Networks \\ Shanghai University, 200072 \\ Shanghai, China \\ Email: cqgan@shu.edu.cn
}

Keywords: virtual passive optical network, multi-subsystem syncretism, metro-access optical network

\begin{abstract}
We propose a novel virtual passive optical network (VPON) scheme syncretizing multiple subsystems for metro-access optical network, which reflects the characteristic of flat network and meets the demand of technology compatibility. In this scheme, in order to maximize the utilization of finite network resource, the threshold-based resource recycling and allocation mechanism supporting the reconfiguration and escalation of VPON is proposed. Finally, we conduct detailed simulations to research and analyze the performance and demonstrate the effectiveness of the proposed scheme.
\end{abstract}

\section{Introduction}

Virtual passive optical network (VPON), as the virtualization of passive optical network (PON), can construct a logically virtual network in the local scope and support open access network[1, 2]. At present, VPON is overall in the primary stage and its research starts from the existing three-layer (core layer, convergence layer and access layer) architecture of optical network[3,4]. On the other hand, the flat network is the inevitable trend of telecommunication network. For the optical network, the flat network will integrate the convergence layer and access layer into the "metro-access layer". Now the architectures and dynamic bandwidth algorithms of metro-access optical network have been widely studied, but the corresponding research on network virtualization has few reports[5, 6]. For example, ref. [5] presents a two-stage time division multiplexing/wavelength division multiplexing metro-access (TDM/WDM) network with all-optical virtual private network (VPN) across different sub-PONs. Ref. [6] proposes an orthogonal frequency division multiplexing (OFDM) -based metro-access network enabling scalable and reconfigurable all-optical VPN.

From the above research on network virtualization of metro-access optical network, we can conclude that current research is carried out in the single subsystem (such as TDM-PON, WDM-PON and OFDM-PON). As network evolution must maintain its smoothness, multi-subsystem syncretism is necessary. Therefore, we propose a novel VPON scheme syncretizing multiple subsystems for metro-access optical network, which reflects the characteristic of flat network and meets the demand of technology compatibility.

\section{A Novel VPON Scheme Syncretizing Multiple Subsystems for Metro-Access Optical Network}

Figure 1 shows a VPON scenario syncretizing multiple subsystems for metro-access optical network, and Figure 2 illustrates the programmable VPON architecture. The network contains a central node (CN), several flexible control nodes (FCN) and traditional optical line terminal (TOLT), and many optical network units (ONU). The CN, FCN and TOLT are distributed in the fiber ring, and each FCN or TOLT with its connected ONUs is in tree topology. The centralized management of the whole network are realized in the $\mathrm{CN}$, which is the unique interface node between core layer and metro-access layer. The FCN, as the intermediate node of the network, has the function of remote node (RN) and OLT, and it includes a wavelength router module and an advanced OLT. The 
wavelength router module can route the wavelength from the CN to the advanced OLT. In addition to, it also can route the wavelength to ONU and achieve the function of $\mathrm{RN}$, and that is forming a WDM-PON in the metro-access network. The advanced OLT is made up of adaptive transceiver, buffer and MAC group. The adaptive transceiver has the adaptability for modulation/demodulation of data streams in different subsystems such as TDM-PON and OFDM-PON, and MAC group can achieve the access control of these subsystems. In summary, multi-subsystem syncretism can be realized by the FCN. Therefore, a VPON can be made up of ONUs in multiple subsystems.

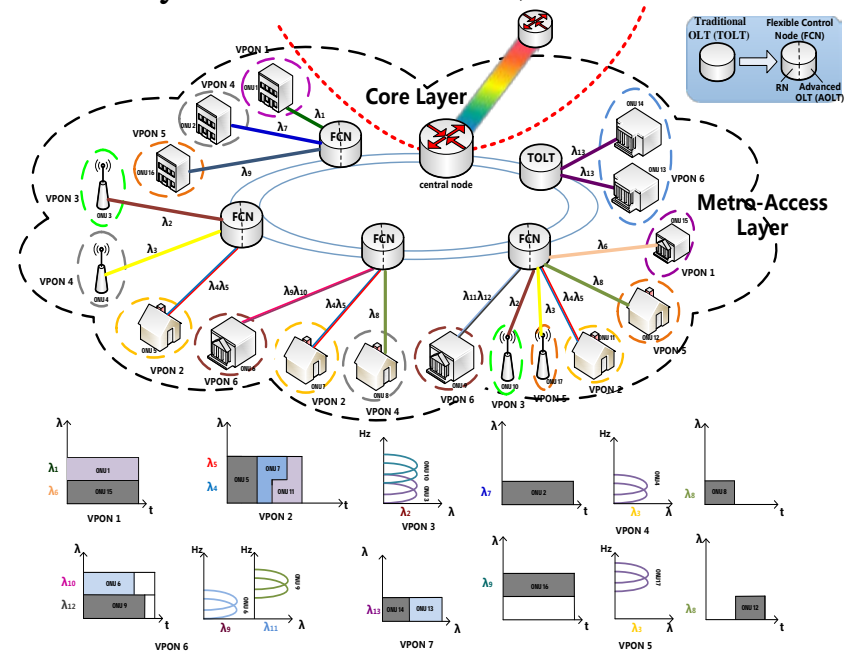

Fig. 1. The VPON scenario syncretizing multiple subsystems

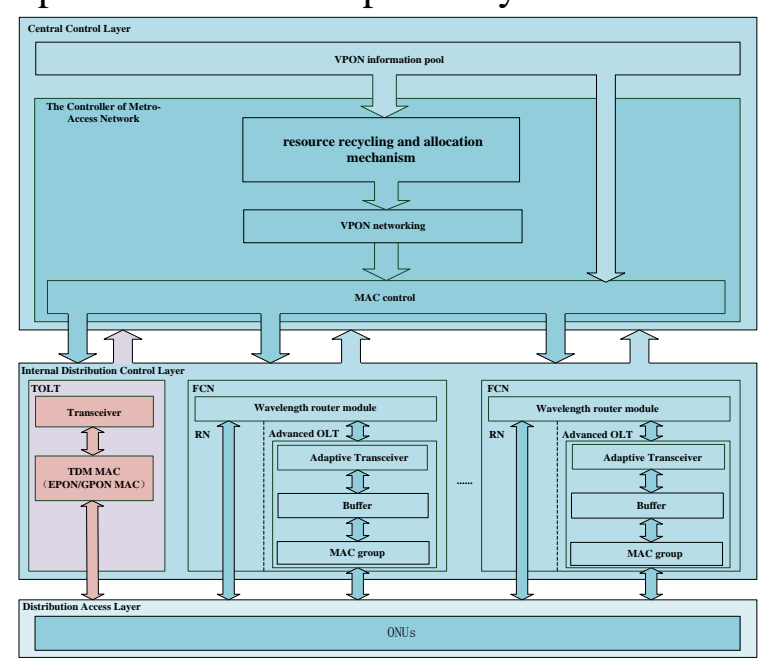

Fig. 2. The programmable VPON architecture

As the reconfiguration or establishment of a VPON need to recycle and allocate network resource, in order to maximize the utilization of finite network resource, the threshold-based resource recycling and allocation mechanism supporting the reconfiguration and escalation of VPON must be employed in this scheme. If a VPON has new demand, it will send requested information to the $\mathrm{CN}$ and it will gather the information to form a VPON information pool. After gathering all the information, the information pool will distribute related information to the controller of metro-access network and the controller will use the information to execute resource recycling and allocation mechanism. For example, if a new VPON want to be admitted, it will send related information to the $\mathrm{CN}$, and the mechanism will be executed to determine whether has enough resource to meet the demand of this new VPON.

\section{Threshold-based resource recycling and allocation mechanism}

The reconfiguration and escalation of a VPON reflect in the change of demand, therefore two sets of VPONs are defined. The first set is VPON's requested bandwidth compared to previous round lies below the low threshold $\eta_{1}$, and the set is denoted by $O_{1}$. The second one is VPON's requested bandwidth compared to previous round lies above the high threshold $\eta_{2}$, and the set is denoted by $\mathrm{O}_{2}$. The flowchart of proposed resource recycling and allocation mechanism is illustrated in Fig. 3. Firstly, we meet the demand of $O_{1}$, and then recycle their excess resource. To allocate resource to $\mathrm{O}_{2}$, we need to figure out its available resource. The available resource consists of two parts: one is the remaining resource of previous round and the other is the resource released by $O_{1}$. After calculating the available resource, we need to classify the $\mathrm{O}_{2}$ into two situations. The first situation is VPONs that already exist in the previous round, and the second one is new VPONs that want to be admitted. This two situations need to be treated differently. For the first situation, we first should maintain their original demand in the previous round, which can ensure fairness for these VPONs, and then figure out additional demand. For the second one, we should figure out the demand requested by the new VPONs. After that, the multi-granularity-based resource allocation algorithm is executed. 


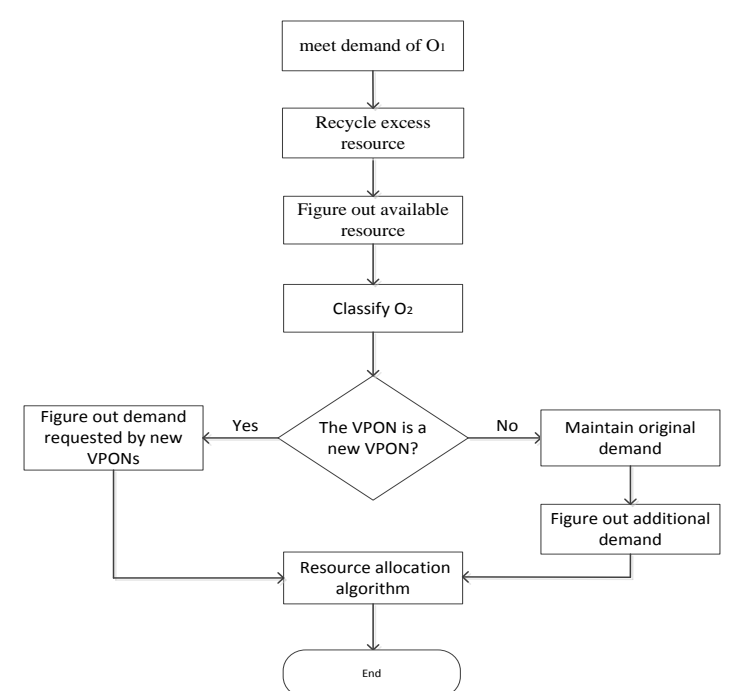

Fig. 3 Flowchart of resource recycling and allocation mechanism

Sort the VPON in a descending order by priority

$\mathrm{T}=\mathrm{S}$

$i=1, j=1$

while $i \leq n$

if $\quad T \geq N(i,:)$

$M(i,:)=\lfloor N(i,:)\rfloor$

$T=T-M(i,:)$

else if $V P O N i \in$ new VPON

if $T \geq G(i,:)$

$M(i,:)=\lfloor G(j,:)\rfloor$

$T=T-M(i,:)$

$j++$

end

end

end

$i++$

Fig. 4 The pseudo-code of the algorithm

In order to illustrate the algorithm, we need to define several matrices here. The available resource is denoted by a matrix $S$

$$
S=\left[\begin{array}{lll}
S_{1} & S_{2} & S_{3}
\end{array}\right]
$$

where $s_{j}, j=1,2,3$ represents the available resource of wavelength, timeslot and subcarrier, respectively.

The remaining resource after each VPON allocation can be denoted by a matrix $T$

$$
T=\left[\begin{array}{lll}
t_{1} & t_{2} & t_{3}
\end{array}\right]
$$

The requested bandwidth of VPONs in a descending order by priority is denoted by a matrix $R$

$$
R=\left[\begin{array}{ccc}
r_{11} & r_{12} & r_{13} \\
r_{21} & r_{22} & r_{23} \\
\vdots & \vdots & \vdots \\
r_{n 1} & r_{n 2} & r_{n 3}
\end{array}\right]
$$

where $n$ is the number of VPONs in the third case, and $r_{i j}, j=1,2,3$ represents VPON $i$ 's requested bandwidth of wavelength resource, timeslot resource and subcarrier resource, respectively. The priority of VPON is defined as the weighted sum of client priority and service priority, and the formula is as follows:

$$
\text { VPON_pri }=\omega_{\text {cli }} \cdot \text { Client_pri }+\omega_{\text {ser }} \cdot \text { Service _ pri }
$$

where $\omega_{c l i}, \omega_{\text {ser }}$ is the weight of client priority and service priority, respectively.

The guaranteed bandwidth of new VPONs is denoted by a matrix $P$

$$
P=\left[\begin{array}{lll}
p_{11} & p_{12} & p_{13} \\
p_{21} & p_{22} & p_{23} \\
\vdots & \vdots & \vdots \\
p_{m 1} & p_{m 2} & p_{m 3}
\end{array}\right]
$$

where $m$ is the number of new VPONs in the third case, and $p_{i j}, j=1,2,3$ represents VPON $i$ 's guaranteed bandwidth of wavelength resource, timeslot resource and subcarrier resource, respectively.

In order to facilitate the calculation, we can convert the requested bandwidth into the number of requested resource, so the matrices $R$ and $P$ can be denoted by matrices $N$ and $G$ 


$$
\begin{aligned}
& N=\left[\begin{array}{lll}
n_{11} & n_{12} & n_{13} \\
n_{21} & n_{22} & n_{23} \\
\vdots & \vdots & \vdots \\
n_{n 1} & n_{n 2} & n_{n 3}
\end{array}\right] \\
& G=\left[\begin{array}{lll}
g_{11} & g_{12} & g_{13} \\
g_{21} & g_{22} & g_{23} \\
\vdots & \vdots & \vdots \\
g_{m 1} & g_{m 2} & g_{m 3}
\end{array}\right]
\end{aligned}
$$

where $n_{i j}=r_{i j} / c_{j}, g_{i j}=p_{i j} / c_{j}$, and $c_{j}, j=1,2,3$ is the resource granularity of wavelength, timeslot and subcarrier, respectively.

The number of resource that a VPON gets finally can be denoted by a matrix $M$

$$
M=\left[\begin{array}{ccc}
m_{11} & m_{12} & m_{13} \\
m_{21} & m_{22} & m_{23} \\
\vdots & \vdots & \vdots \\
m_{n 1} & m_{n 2} & m_{n 3}
\end{array}\right]
$$

where $m_{i j}, j=1,2,3$ represents the number of wavelength, timeslot and subcarrier resource that a VPON gets after resource allocation algorithm.

The pseudo-code of the proposed algorithm is presented in Fig. 4. As shown in Fig. 4, this algorithm can improve the number of admitted VPONs because the new VPONs can be admitted as long as their guaranteed bandwidth is satisfied.

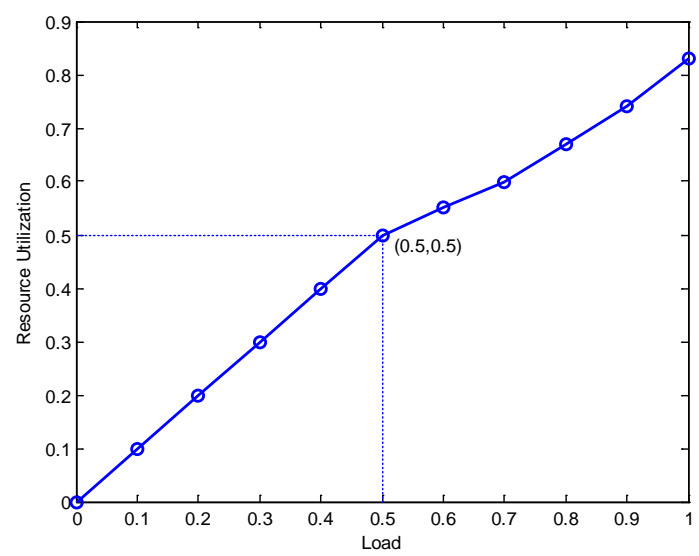

Fig. 5 The simulation of resource utilization

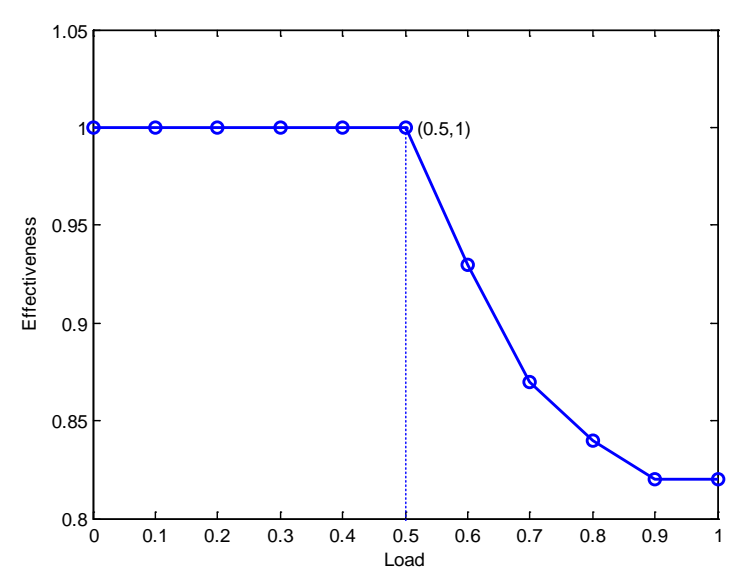

Fig. 6 The simulation of effectiveness

\section{Performance Evaluation}

In this section, we use Matlab to evaluate the performance of proposed resource allocation algorithm in terms of resource utilization and effectiveness. The resource utilization of proposed scheme is depicted in Fig. 5. As shown in Fig. 5, the resource utilization is proportional to the network load when the load is below 0.5. However, as the load increases, the demand of some VPONs can be not fully met, so the resource utilization is not proportional to the network load any longer, and the resource utilization is 0.82 when the load is 1 . The effectiveness of proposed scheme is depicted in Fig. 6. The effectiveness is always one when the network load is below 0.5, which means the demand can always be met, and the result is consist with that in Fig. 5 . When the load increases, the effectiveness decreases dramatically because the resource can not fully meet the demand of VPONs. However, when the load is above 0.9, the effectiveness is almost unchanged 
because the remaining resource can only ensure the guaranteed bandwidth of new VPONs instead of fully meeting their demand.

\section{Conclusion}

A novel VPON scheme syncretizing multiple subsystems for metro-access optical network is proposed. In this scheme, a VPON is made up of ONUs in multiple subsystems. As the reconfiguration or establishment of a VPON need to recycle and allocate network resource, in order to maximize the utilization of finite network resource, the threshold-based resource recycling and allocation mechanism supporting the reconfiguration and escalation of VPON is introduced in this scheme. Based on this mechanism, we propose the multi-granularity-based resource allocation algorithm which can improve the number of admitted VPONs. The simulation result and analysis show a good performance of our proposed algorithm in terms of resource utilization and effectiveness.

\section{Acknowledgements}

This work is supported by Programs of National Science Foundation of China (No. 61132004, 61275073 and 61420106011), Shanghai Science and Technology Development Funds (No. 13JC1402600 and 14511100100), Shanghai Leading Academic Discipline Project (No. S30108), Shanghai Economics and Information Project (No. CXY-2013-93) and Key Laboratory of Specialty Fiber Optics and Optical Access Networks, Shanghai University (No. SKLSFO2012-05).

\section{References}

[1] W. Wei, J. Hu, D. Qian et al. PONIARD: A Programmable Optical Networking Infrastructure for Advanced Research and Development of Future Internet. Journal of Lightwave Technology, vol.27, no.3, Feb. 2009, pp: 233-242.

[2] L. Zhou, H. Lin, G. Peng et al. A Novel Optical Access Network: Software-Defined FlexPON. OECC/ACOFT 2014, pp: 688-690.

[3] X. Hu, L. Zhang, P. Cao et al. Reconfigurable and Scalable All-Optical VPN in WDM PON. IEEE Photonic Technology Letters, vol. 23, no. 14, July 2011, pp: 941-943.

[4] Z. Zhang, M. Xu, J. Wu et al. An OFDMA-PON architecture supporting flexible all-optical VPN with source-free ONUs. PGC 2012, pp: 1-3.

[5] Y. Tian, Q. Chang and Y. Su. A Two-Stage Metro-Access Integrated Network Enabling All-Optical Virtual Private Network. ECOC 2008, pp: 1-2.

[6] W. Jin, C. Zhang et al. Scalable and Reconfigurable All-Optical VPN for OFDM-Based Metro-Access Integrated Network. Journal of Lightwave Technology, vol. 32, no.2, Jan. 2014, pp: 318-325. 